Carnets de géographes

GÉOGRAPHES

\title{
Le nouveau paysage religieux de la banlieue parisienne
}

L'exemple de la communauté d'agglomération «Val de France »

Hervé Vieillard-Baron

\section{(2) OpenEdition}

\section{Journals}

Édition électronique

URL : http://journals.openedition.org/cdg/923

DOI : $10.4000 /$ cdg.923

ISSN : 2107-7266

\section{Éditeur}

UMR 245 - CESSMA

\section{Référence électronique}

Hervé Vieillard-Baron, «Le nouveau paysage religieux de la banlieue parisienne », Carnets de géographes [En ligne], 6 | 2013, mis en ligne le 01 septembre 2013, consulté le 08 janvier 2020. URL : http://journals.openedition.org/cdg/923 ; DOI : 10.4000/cdg.923

Ce document a été généré automatiquement le 8 janvier 2020.

\section{c) (†) $९$}

La revue Carnets de géographes est mise à disposition selon les termes de la Licence Creative Commons Attribution - Pas d'Utilisation Commerciale - Pas de Modification 4.0 International. 


\title{
Le nouveau paysage religieux de la banlieue parisienne
}

\author{
L'exemple de la communauté d'agglomération « Val de France »
}

Hervé Vieillard-Baron

\section{Introduction}

1 Depuis la fin du XIXème siècle, on a coutume d'associer la croissance urbaine au déclin de la religion, comme si le développement des villes et les migrations qui l'accompagnent allaient de pair avec la sécularisation et la décomposition des anciennes appartenances (Endelstein, Fath et Mathieu, 2010). En réalité, le religieux ne se dissout pas dans les tours et les barres des grands ensembles. Avec les migrations et la croissance du pluralisme religieux, on observe à la fois une recomposition des anciennes croyances et une réaffirmation des cultures d'origine. Précisément, les quatre communes de la grande couronne parisienne qui se sont associées en 1997 pour former la communauté d'agglomération «Val de France », située au sud du Val d'Oise, sont un reflet significatif de cette situation. En une soixantaine d'années, Arnouville, Garges-lès-Gonesse, Sarcelles et Villiers-le-Bel ont vu leur population quintupler et leur territoire s'urbaniser massivement. Répondant à une politique d'ouverture jusqu'au milieu des années 1980, l'arrivée successive de strates migratoires issues de tous les continents a profondément transformé les sociétés locales. Diverses formes de marquages communautaires (édifices religieux, monuments commémoratifs, locaux associatifs, écoles, commerces, services divers à l'usage des migrants...) ont progressivement vu le jour, imposant dans certains quartiers une nouvelle régulation de la diversité. La cartographie des lieux de culte a été complètement renouvelée et les modèles hérités des pratiques catholiques, considérés longtemps comme majoritaires, sont devenus minoritaires.

2 Du point de vue méthodologique, l'étude présentée ici s'appuie d'une part sur deux recensements, celui des lieux de culte actualisé en mars-avril 2012 avec l'aide de la mission "Mémoires et identités en Val de France », des associations concernées et des municipalités, et celui effectué par l'Insee en 2008, d'autre part sur des enquêtes semi- 
directives menées auprès de divers responsables associatifs et de quelques anciens habitants, et sur des travaux menées antérieurement, notamment sur le grand ensemble de Sarcelles-Lochères (Vieillard-Baron, 1992). Les limites de l'analyse tiennent non seulement à l'absence d'indications concernant l'appartenance religieuse dans les recensements et à la forte mobilité des ménages, mais aussi à l'augmentation des pratiques déterritorialisées (dans le cadre des églises évangéliques en particulier) et à la volatilité des communautés qui ont saisi une opportunité provisoire pour s'installer sur le territoire de l'agglomération. Par exemple, les nouveaux mouvements religieux (les pentecôtistes notamment) se contentent souvent de locaux précaires parce qu'ils ne recrutent pas leurs membres en référence à un lieu, mais à partir de réseaux de connaissance et de sensibilité (Dejean, 2010). Le risque serait donc de proposer un tableau rigide de l'inscription spatiale des lieux de culte alors qu'une partie d'entre eux sont mobiles, sinon en construction ou en restructuration, voire même en situation d'abandon.

\section{Une progression remarquable et une extrême diversité des lieux de culte}

3 Selon le recensement effectué en 2012 à Sarcelles, Villiers-le-Bel, Arnouville et Garges-lèsGonesse, le nombre de lieux de culte a décuplé dans le périmètre de l'agglomération en une centaine d'années, et quadruplé en quarante ans: on est passé ainsi de 4 lieux de culte en 1900 (une église catholique dans chaque commune) à 13 lieux de culte en 1965 et à 59 en mai 2012 (70 en incluant les églises évangéliques non affiliées, les lieux de culte en construction et ceux dont le permis de construire a été déposé).

Figure : Évolution du nombre de lieux de culte dans les communes de l'agglomération

\begin{tabular}{|c|c|c|c|}
\hline \multirow[b]{2}{*}{ Communes concernées } & \multicolumn{3}{|c|}{ Nombre de lieux de culte } \\
\hline & 1900 & 1965 & 2012 \\
\hline Arnouville & 1 & 4 & 8 \\
\hline Garges-lès-Gonesse & 1 & 2 & 14 \\
\hline Sarcelles & 1 & 4 & 22 \\
\hline Villiers-le-Bel & 1 & 3 & 15 \\
\hline Total Agglomération «Val de France » & 4 & 13 & 59 \\
\hline
\end{tabular}

4 Les trois-quarts de ces lieux de culte ont été créés par et pour les migrants ${ }^{1}$, chaque groupe d'originaires apportant sa propre pluralité religieuse à la société locale, même si l'on observe des regroupements de mouvances historiquement proches avec la migration et l'installation dans le pays d'accueil. A titre d'exemple, les chrétiens assyro-chaldéens qui peuvent être catholiques orientaux uniates (c'est-à-dire unis au pape de Rome), nestoriens, jacobites ou orthodoxes syriaques, ont abandonné une grande partie de leurs différences en se trouvant réunis à Sarcelles par les hasards de la migration. Dans un autre registre, les Indiens de Pondichéry peuvent être musulmans, catholiques, 
protestants, hindouistes, sikhs ou bouddhistes, mais ils ont tendance à se définir en France par leur identité pondichérienne, en relation avec l'ancien comptoir français. Stratégiquement, dans les négociations avec les collectivités territoriales (les municipalités en particulier), ces migrants ont compris qu'il était préférable de se présenter comme une communauté rassemblée, au moins dans les premières années qui suivent l'installation sur le territoire français.

5 Pour mémoire, on rappellera ici que le phénomène migratoire a été le moteur principal de la croissance de la banlieue nord de Paris et qu'il est constitutif des identités locales depuis l'installation des premiers Arméniens à Arnouville dans le département de Seineet-Oise en 1922 jusqu'aux Pakistanais et Comoriens dernièrement arrivés. En ce qui concerne Sarcelles, le recensement de l'Insee indiquait en 1990 que 39 \% des Sarcellois étaient nés hors de métropole, $46 \%$ pour le seul grand ensemble de Sarcelles-Lochères.

6 En 2008, l'Insee comptabilisait 28032 étrangers, soit $20 \%$ des 140000 habitants des quatre communes ${ }^{2}$. Mais avec les réintégrations dans la nationalité, l'apport des naturalisations, l'acquisition de la nationalité française et la naissance sur place des deuxième et troisième générations, les édiles locaux estiment couramment que près de la moitié de la population totale des quatre communes est aujourd'hui issue de l'immigration. En 2008, un recensement des titres de séjour effectué pour le compte de la communauté d'agglomération mettait en évidence l'existence de 129 nationalités 3 .

7 L'affirmation de la diversité culturelle actuelle dépend non seulement des migrations récentes, mais aussi, par effet mécanique, de l'enracinement de ménages issus de l'immigration et ayant conservé une fécondité supérieure à la moyenne française. A partir des données du recensement de 1999, la démographe Michèle Tribalat observait déjà que Garges comptait $66,5 \%$ de " jeunes d'origine étrangère ${ }^{4}$, Sarcelles $62,2 \%$ et Villiers le Bel 58,9 \%, ces trois villes étant respectivement les deuxième, huitième et onzième villes de France pour leur proportion de jeunes d'origine étrangère (Tribalat, 2006). Dans la communauté d'agglomération, on constate que la proportion de jeunes de moins de 18 ans d'origine maghrébine a plus que doublé de 1968 à 1999, et que celles des jeunes d'origine turque et subsaharienne, quasiment nulle en 1968, a atteint près de $10 \%$ des moins de 18 ans.

8 Il va de soi que le nombre des édifices religieux et des implantations cultuelles ou culturelles traduisent cette multiplicité des origines. En 2012, on dénombrait ainsi sur le territoire de la communauté d'agglomération, 9 églises ou chapelles catholiques romaines, 5 églises orientales (deux arméniennes, une copte et deux églises chaldéennes dont une en construction à Arnouville), un temple réformé, une quinzaine d'églises évangéliques (dont 3 églises baptistes) ${ }^{5}$, une église néo-apostolique, 23 synagogues ou oratoires juifs ${ }^{6}, 12$ salles de prières musulmanes (dont une mosquée en construction à Garges-lès-Gonesse et une grande mosquée dont le permis de construire aurait été déposé à Sarcelles), une pagode bouddhiste (d'origine lao) et une salle du Royaume dédiée aux Témoins de Jéhovah.

9 Si l'on met à part les églises catholiques dont la construction est souvent antérieure à la loi de séparation des Églises et de l'État de 1905 (et qui bénéficient à ce titre d'avantages importants dont l'entretien des bâtiments par les services municipaux), l'implantation des lieux de culte est étroitement associée à la chronologie de l'histoire coloniale et de la décolonisation, mais aussi aux conditions économiques et sociales de l'immigration. Pour en comprendre les logiques de localisation, il faut tenir compte des conditions d'accueil 
offertes par les municipalités, du marché du travail à l'époque de la migration, de l'offre foncière et, plus encore, de l'offre locative à destination de nouveaux ménages souvent très modestes - aussi bien dans le parc social HLM que dans le parc social « de fait ». A Sarcelles et à Garges, plus de $50 \%$ du parc de logements est de type HLM $(52,7 \%$ à Sarcelles et 51,3 \% à Garges en 1999), sans compter les copropriétés « sociales ».

Il est légitime de penser que la localisation des premiers arrivés et la publicité qu'ils ont pu en donner dans leurs réseaux ont permis, à des degrés divers, la constitution de filières. De multiples formes de regroupements communautaires ont pu se constituer ainsi sur des bases familiales, affinitaires ou religieuses.

11 En ce qui concerne l'église catholique, l'origine nationale des prêtres nouvellement nommés et la localisation géographique de leur affectation précédente constituent également un facteur d'appel. Par exemple, un prêtre de retour de mission au Vietnam a attiré beaucoup de Vietnamiens dans l'église Saint-Martin du vieux pays de Garges où il venait d'être nommé. De la même façon, un prêtre d'origine congolaise (RDC) a fait venir de nombreux congolais du Val d'Oise après sa nomination dans cette même église.

Quant à la paroisse Sainte Geneviève, située à Garges également, elle comptait en 2005 trois diacres étrangers : un vietnamien, un cap-verdien et un congolais. Il faut dire que la communauté catholique est très diversifiée dans cette ville avec plus de cinquante nationalités et que le travail en réseau s'avère nécessaire, ne serait-ce que pour dépasser la barrière de la langue. A l'occasion de son installation, le nouveau curé a organisé un grand repas œcuménique regroupant notamment le rabbin, le président de la communauté musulmane de Garges et des fidèles des principales religions.

Cette ouverture à la diversité culturelle se rencontre dans les autres communes, en particulier à Sarcelles où l'un des prêtres interrogé au printemps 2009 déclarait :

"Chaque dimanche, nos églises, surtout celle du Bienheureux Jean XXIII, prennent

l'allure d'une véritable usine du Bon Dieu. Après la messe de 8 heures, il y a celle de 9 heures pour les Sri-Lankais ou les Vietnamiens, puis, après celle de 10 heures 30 qui est pour tous, celle de 13 heures pour les Chaldéens, celle-ci précédant l'assemblée du Renouveau charismatique... ».

De leur côté, les églises protestantes - qui sont pour la plupart des églises évangéliques ont tendance à se renforcer par l'hébergement d'églises plus modestes, ou par un soutien actif à l'essaimage de communautés dans d'autres communes. Ainsi, l'église réformée de Sarcelles ("le Grenier») a accueilli dans le grand ensemble de Lochères une église camerounaise pendant plusieurs années. De même, l'église «Source de Vie » qui s'est implantée à Sarcelles en 1985 et qui appartient au réseau d'églises protestantes évangéliques de la banlieue nord de Paris ${ }^{7}$, héberge une église pour malentendants et une église tamoule. Le pasteur leur a prêté des locaux et a aidé les responsables à rédiger les statuts de leur association.

A partir de cette église-mère "Source de Vie », six « églises-filles » sont ont vu le jour, portées par l'arrivée de nouveaux Antillais et de migrants d'origine étrangère. Par exemple, l'une d'entre elles, d'origine franco-haïtienne, après avoir été hébergée à Goussainville, a loué une salle dans l'hôtel Ibis de Sarcelles avant de trouver des locaux plus stables à Arnouville, en l'occurrence une ancienne scierie aménagée en salle de prière. Selon le pasteur haïtien qui en est le responsable, 150 fidèles fréquentent cette "église " très régulièrement. Les chants ont évolué avec l'introduction de nouveaux instruments de musique et de rythmes afro-antillais. En somme, l'église «Source de vie » 
s'est adaptée à la diversité culturelle, non sans irriter quelques anciens pratiquants, il est vrai.

\section{Une inscription inégale dans l'espace}

16 Les lieux de culte créés par les migrants s'inscrivent dans l'espace selon la dialectique récurrente "visibilité/invisibilité ». Si l'on met à part les édifices catholiques, certains affichent leur caractère religieux, d'autant plus ouvertement qu'ils ont été aidés par des financements extérieurs à la communauté locale (églises catholiques, églises arméniennes d'Arnouville, église chaldéenne de Sarcelles, synagogues consistoriales de Sarcelles et de Garges...). D'autres lieux de culte sont, au contraire, totalement invisibles: ils sont souvent implantés dans des locaux qui ont été détournés de leur fonction initiale (appartements, pavillons, locaux commerciaux, espaces industriels, salles d'hôtels ou de réception, espaces autrefois consacrés à une autre pratique religieuse...).

17 En systématisant les choses, on peut distinguer trois types de lieux correspondant chacun à des besoins spécifiques, en corrélation avec leur puissance d'attraction :

18 - le lieu de proximité qui se fait en général discret et qui relaie les besoins des pratiquants du quartier, la relative invisibilité du local reflétant souvent la précarité dans laquelle se trouvaient les fidèles à leur arrivée. Si l'on met à part les paroisses catholiques qui ont une tradition de visibilité, tout au moins avant la guerre de 1939-45, la majorité des salles de prières musulmanes, le temple réformé de Sarcelles («Le Grenier ») et la plupart des oratoires juifs sont dans ce cas.

19 - le lieu-source qui attire bien au-delà du quartier et qui sert de regroupement pour tous les fidèles de la région, avec l'objectif de manifester le poids et l'unité de la communauté. L'église copte de Sarcelles, la pagode bouddhiste de Villiers-le-Bel, le centre loubavitch Beth Habad de Sarcelles, l'église néo-apostolique majoritairement congolaise, les écoles juives, certaines synagogues et une bonne partie des églises évangéliques (dont l'église sarcelloise "Source de Vie », ou encore les églises de Réveil congolaises) sont concernées.

20 - le lieu-symbole qui exprime son ancrage dans la cité par des attributs architecturaux bien visibles (clocher, minaret ou coupole, chandelier à huit ou neuf branches...), mais qui peut se présenter également comme un lieu-source et un lieu de proximité. C'est le cas par exemple des anciennes églises catholiques des quatre communes concernées (notamment Saint-Martin de Garges et Saint-Pierre-Saint-Paul de Sarcelles construite au $13^{\text {ème }}$ siècle sur un ancien temple païen et remaniée au $16^{\text {ème }}$ siècle), de l'église arménienne Sainte-Croix de Varak à Arnouville, mais aussi de la synagogue consistoriale inaugurée en 1965 à Sarcelles, de l'église chaldéenne Saint-Thomas construite à proximité de la gare de Sarcelles-SaintBrice et consacrée en 2004. La future grande mosquée de Sarcelles, si l'on en croit le site internet à son propos $^{8}$ devrait être également un futur lieu-symbole si elle est effectivement construite avec deux grands minarets, à proximité de la gare de GargesSarcelles. 
Figure : La mosquée « Foi et unicité » d'inspiration sunnite à Sarcelles

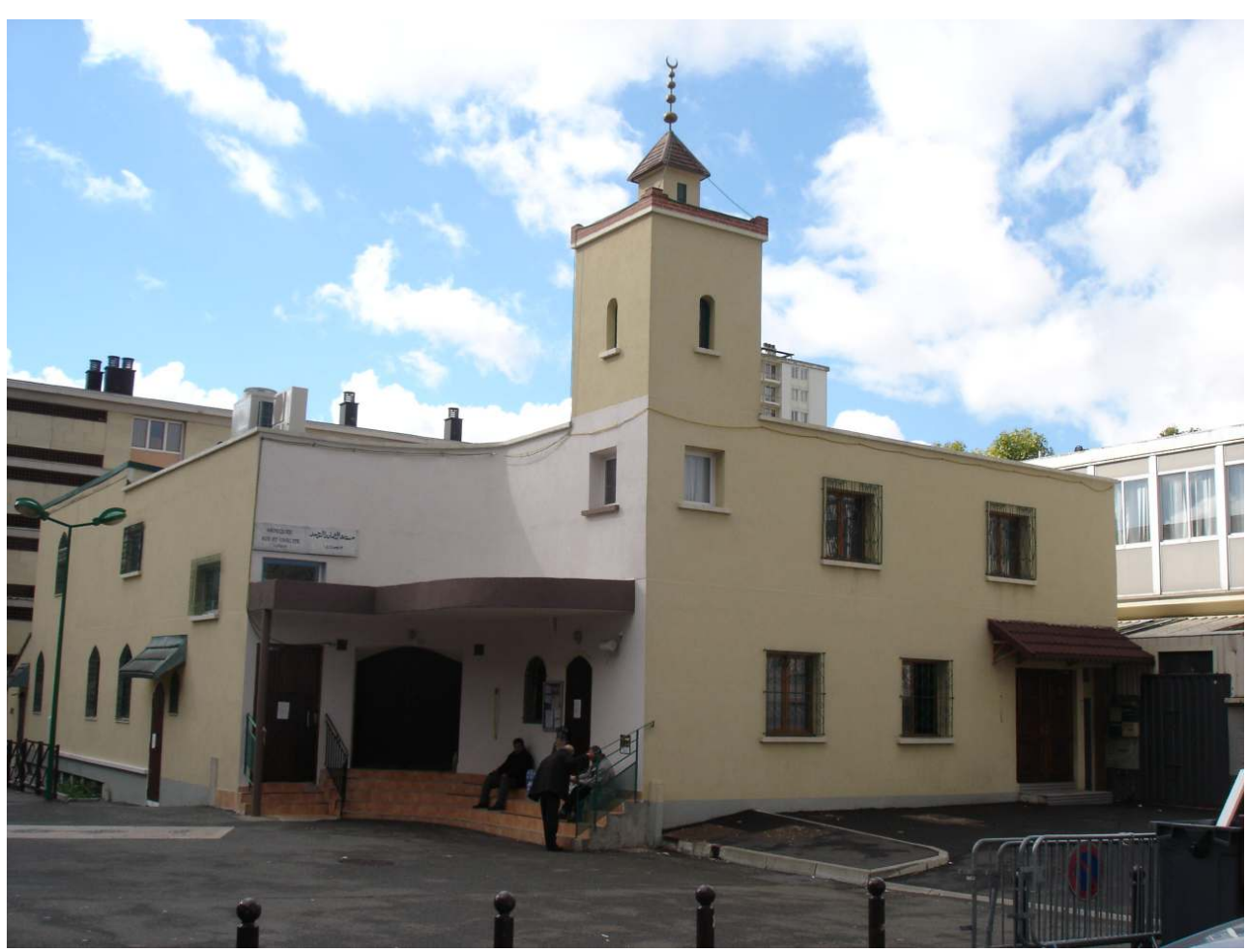

cliché : Hervé Vieillard-Baron, mai 2011

21 Les enquêtes effectuées localement montrent que chaque lieu de culte régulièrement fréquenté assume une diversité de fonctions : fonction cultuelle associé à l'exercice du culte et à l'éducation religieuse, fonction culturelle avec l'ouverture d'une bibliothèque, la présence de cours de danse, de sport ou de musique, et l'organisation d'expositions ou de conférences pour assurer la diffusion de la culture religieuse, fonction de formation avec des cours de langue, des enseignements de base sur les écrits fondamentaux et des cours spécialisés sur des notions et des textes plus pointus, fonction sociale pour soutenir les familles en difficulté, venir en aide aux plus démunis et coordonner les activités bénévoles (soutien scolaire, garderie, repas, fêtes, etc.). Il ne faudrait pas négliger non plus les opportunités financières que peuvent constituer certains services communautaires (enseignement de l'arabe coranique, vente de vêtements rituels par exemple), ni les structures commerciales qui y sont parfois associées, celles-ci pouvant expliquer la démultiplication des lieux de culte dans la communauté d'agglomération.

22 Ainsi, chaque religion tend à répondre tout à la fois au souci spirituel et au souci temporel des croyants en les associant directement à la transmission des valeurs propres à la communauté, le lieu de culte devenant un centre de vie sociale et un espace de ressourcement communautaire. On pourrait parler de «lieu-providence», comme on parle « d'église-providence » à propos des Evangéliques. 
Figure 3 :L'église chaldéenne Saint Thomas inaugurée en 2004 à Sarcelles

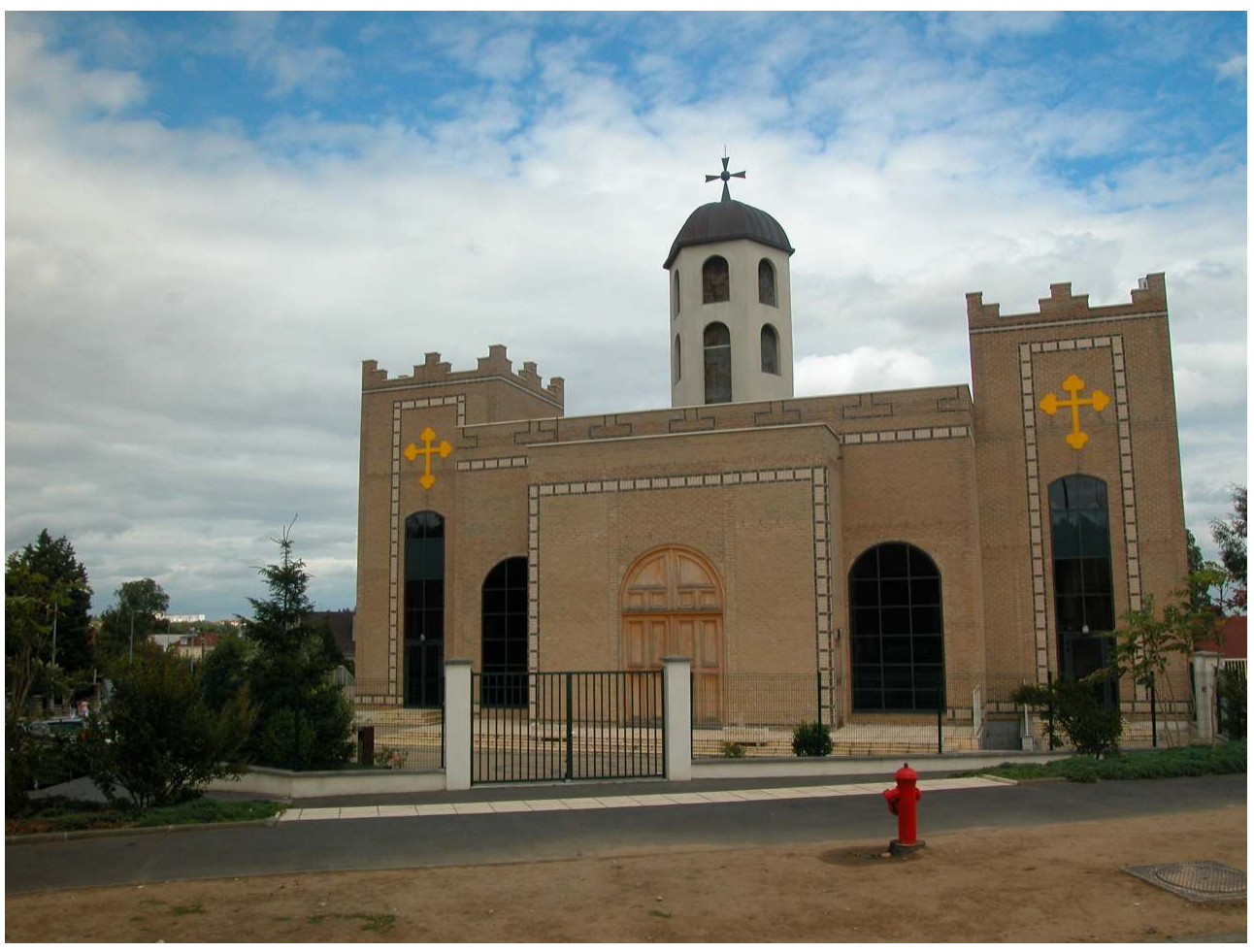

cliché : Hervé Vieillard-Baron, mai 2012

\section{Une mémoire qui perdure en dépit des fractionnements communautaires}

L'extrême variété des territorialisations religieuses résulte autant de la revendication d'une foi et d'une mémoire partagées que d'une somme d'histoires singulières. Elle dépend de facteurs locaux liés aussi bien à l'accueil des institutions religieuses représentatives, au positionnement des élus, à la sensibilité du voisinage, au fractionnement interne des communautés, qu'à la personnalité des responsables associatifs ou des ministres du culte (par exemple le rabbin Guedj ou le Rav Israel qui a ouvert un centre d'études de la thora - kollel -en 1975 à Sarcelles). Le grand nombre de synagogues provient d'une distance plus ou moins marquée au Consistoire, de la nature des enseignements professés (cours sur le Tamud), mais surtout de la multiplicité des origines micro-géographiques des pratiquants, chacun d'entre eux restant sensibles non seulement à sa ville et à son quartier d'origine (Constantine, Alger, Oran, Tlemcen, Le Caire, Alexandrie, Tanger, Mogador, Mazagan, Fès, Tunis, Djerba...), mais aussi à la mélodie de son ancienne synagogue. En 2012, rien qu’à Sarcelles, on dénombrait 17 synagogues ou oratoires. La grande synagogue, en cours d'extension, est elle-même subdivisée : elle abrite les Algériens au rez-de-chaussée, les Tunisiens au premier étage et les Marocains au second étage. Mais le grand nombre de synagogues ne signifie pas un enracinement définitif des fidèles. Depuis la naissance du grand ensemble, les juifs se sont largement redistribués dans la région parisienne. Si beaucoup d'entre eux ont déménagé à Paris ou vers les communes voisines de Sarcelles en accédant à la propriété (tout particulièrement à Saint-Brice), le renouvellement s'est opéré grâce à la notoriété de Sarcelles comme centre actif de services communautaires au sein du judaïsme français et dans les réseaux mondiaux. 

de France sont également fractionnés ${ }^{9}$, mais leurs lieux de culte se sont développés en décalage temporel avec l'implantation géographique des ménages. Compte tenu du retard des débuts, on assiste aujourd'hui à de multiples formes de rattrapage. Les projets de mosquée se sont multipliés depuis une quinzaine d'années émanant aussi bien d'anciennes associations ${ }^{10}$ que de nouveaux venus cherchant à manifester leur présence : Turcs, Africains subsahariens, Indiens, Bengalis, Pakistanais, Comoriens par exemple. Il faut dire que les locaux loués ou prêtés par les municipalités, par les organismes de logement ou d'autres institutions religieuses n'étaient souvent pas adaptés aux besoins : cave, appartement, salle de boxe, salle de télévision dans les foyers, entrepôt industriel, antenne jeunesse.

Un des problèmes soulevé localement est la fréquentation très irrégulière dans la journée et très variable dans le temps, des mosquées et salles de prière. Le document «fréquentation de la mosquée El Irshad» rédigé par l'association gestionnaire en avril 2011 souligne bien cet aspect ${ }^{11}$. Si l'on exclut le vendredi, les prières du matin accueillent une dizaine de fidèles tandis que celles du soir regroupent une soixantaine d'hommes, les femmes étant généralement absentes. Quant à la prière du vendredi soir, elle regrouperait en moyenne 700 hommes et 150 femmes. Les prières à l'occasion des fêtes de l'Aïd pourraient rassembler jusqu'à 800 hommes et 400 femmes. Cette sur-occupation des locaux, quoiqu'éphémère, a conduit à la conception d'un nouveau projet de mosquée comprenant une salle pour les femmes de $220 \mathrm{~m}^{2}$ pouvant accueillir jusqu'à 440 personnes, et une salle pour les hommes de $420 \mathrm{~m}^{2}$ susceptible de recevoir 840 personnes environ. Les salles de cours et la bibliothèque resteront en l'état, mais une librairie devrait être ouverte.

Figure 4: Local de la mosquée El Irshad à Garges-lès-Gonesse

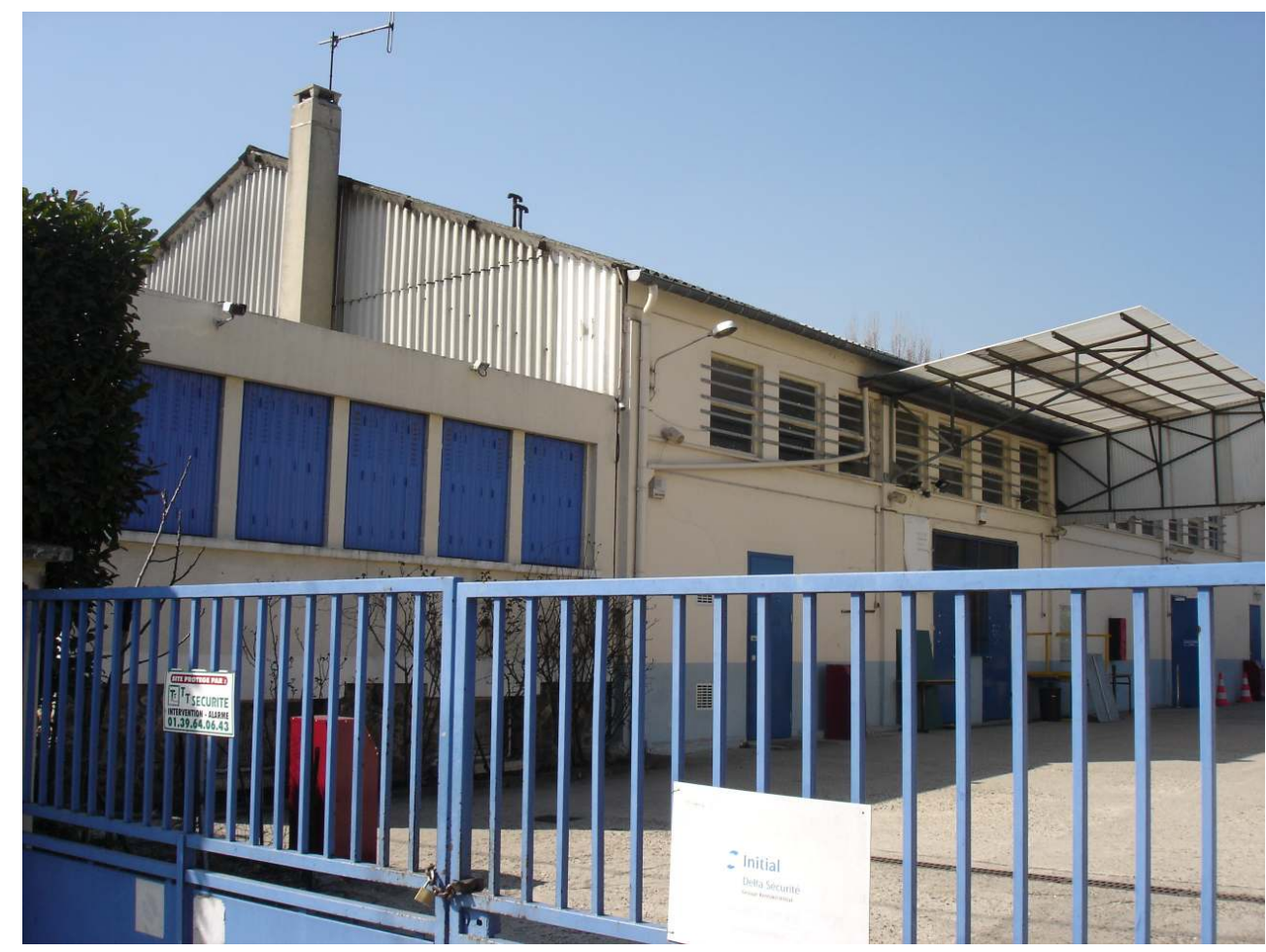

cliché : Hervé Vieillard-Baron 2011.

Carnets de géographes, 6 | 2013 
Quant aux églises évangéliques qui ne sont pas enracinées, elles utilisent la plupart du temps des locaux provisoires : hôtel, espace vacant dans les zones industrielles, magasin désaffecté, garage, ancienne boîte de nuit... Néanmoins, ce sont ces regroupements qui apparaissent aujourd'hui les plus dynamiques du point de vue de la fréquentation et du prosélytisme. Selon le Pasteur Jean-Claude Boutinon, ancien professeur de biologie et aujourd'hui responsable de l'église «Source de vie " à Sarcelles, on ne compterait pas moins de 300 églises évangéliques en 2012 dans les départements de Seine-Saint-Denis et du Val d'Oise. Rien qu'à Saint-Denis, dans le secteur de La Briche, on dénombre une trentaine de salles louées le dimanche matin pour les offices évangéliques (Dejean, 2010). Qu'on ne s'y trompe pas, ce foisonnement est significatif de l'extrême fractionnement de ces églises et $\mathrm{du}$ souci d'indépendance de leurs fidèles, souci exprimé tout particulièrement par les églises tamoules sur le territoire de Val de France. ${ }^{12}$

Au-delà des églises chrétiennes, on observe dans les quatre communes de l'agglomération un renouveau expansionniste des pratiques qui peut s'accompagner d'une radicalisation. De nombreux lieux de culte (mosquées, églises, synagogues...) sont décrits par les officiants comme " pleins à craquer » et l'on observe parfois un empiétement sur l'espace public, en particulier lors des fêtes juives, des offices évangéliques et des prières musulmanes du vendredi.

Cette montée du religieux est illustrée par le nombre élevé des déclarations des associations à caractère cultuel et culturel enregistrées à la sous-préfecture de Sarcelle. Ainsi 35 associations à caractère religieux dont le siège social est situé sur le territoire de Val de France ont été enregistrées à la sous-préfecture de Sarcelles depuis 1999, la plupart d'entre elles se réclamant d'une église évangélique, certaines d'entre elles affirmant une dimension nationale ou ethnique (ivoirienne, camerounaise, congolaise, béninoise, haïtienne, caraïbe et tamoule par exemple) ${ }^{13}$.

\section{Des quartiers inégalement marqués par les pratiques religieuses}

30 Si les modalités d'insertion des lieux de culte dans un quartier influencent les pratiques, il n'est pas rare que celles-ci modifient en retour la vie du quartier. A Sarcelles, le secteur des Cholettes où les commerces casher et les services destinés aux juifs pratiquants sont nombreux est parfois dénommé «Petite Jérusalem». Depuis la naissance du grand ensemble, et au fil des tranches de construction de 1956 à 1975, on y observe, à partir d'un ancien noyau ashkénaze, un regroupement progressif des juifs séfarades issus des pays du Sud de la Méditerranée, tunisiens et algériens en particulier, égyptiens et marocains à un moindre degré. On considère parfois, à partir des noms relevés sur les boîtes aux lettres des immeubles, que ce secteur des Cholettes constitue aujourd'hui un véritable «territoire ethno-religieux» (Benveniste, 1999). Laurence Podselver, chercheure à l'EHESS, souligne la continuité des espaces entre la synagogue, les commerces casher, le mémorial de la Shoah et l'école orthodoxe Torat Emet. Elle montre aussi que l'avenue Paul Valéry, dans ce même secteur, constitue un espace de rencontre où les jeunes juifs mettent en scène par des signes visibles leur appartenance à la communauté. C'est, en quelque sorte, ce rapport fluctuant aux frontières entre le public et le privé, avec des formes temporaires d'appropriation de l'espace, en mimétisme avec la ville israélienne, qui fait le quartier juif. Les réseaux y sont très dynamiques : qu'il s'agisse de la location ou 
de l'achat d'un appartement, d'une école ou d'un service spécifique, l'information y circule très rapidement par les circuits familiaux ou le jeu des interconnaissances. Compte tenu des mobilités résidentielles, ce quartier fonctionne comme un «sas rassurant " pour beaucoup de familles; il constitue un espace de transition marqué tout à la fois par le souvenir du pays d'origine, l'accès direct aux services communautaires et l'acculturation aux valeurs de la République (Podselver, 2000).

Les Assyro-chaldéens qui sont arrivés à Sarcelles pour la plupart dans les années 1980 en provenance de la province de Hakkari à l'extrême est de la Turquie, ont également constitué une sorte de quartier chaldéen en se regroupant dans le lotissement des Chardonnerettes où plus d'une centaine de pavillons leur appartiennent. Alors qu'ils ont été dispersés à leur arrivée en fonction des opportunités locatives au sein du grand ensemble, ils ont pu progressivement accéder à la propriété. L'entraide communautaire, le regroupement de deux ou trois familles, le petit pécule rapatrié de Turquie ou d'Irak, et surtout les économies réalisées au prix d'un travail acharné dans les premières années d'installation leur ont permis de disposer de l'apport initial indispensable. Mais leur regroupement est moins visible que celui des séfarades, d'autant que le grand projet qui les a mobilisés dix années durant, à savoir la grande église chaldéenne Saint-Thomas, finalement consacrée en 2004, se situe à la frontière des communes de Sarcelles et de Saint-Brice, assez loin de leurs lieux d'habitation.

En revanche, d'autres quartiers ne sont que faiblement marqués par la présence des nouveaux acteurs religieux, l'évolution massive de la culture du réseau ne se traduisant pas obligatoirement dans l'espace. A l'exception de la mosquée «Foi et Unicité » de Sarcelles (figure 2), les mosquées ne sont pratiquement pas visibles sur le territoire de l'agglomération. Néanmoins, pour éviter la dispersion de leurs lieux de culte et leur donner plus de visibilité, les musulmans de Garges envisagent la construction d'une grande mosquée fédératrice.

Globalement, les problèmes les plus souvent soulignés lors des entretiens concernent la circulation, les accès aux parkings et le stationnement des véhicules pendant les offices. En ce qui concerne la mosquée El Irshad de Garges, une organisation a été mise en place avec l'aide d'une trentaine de bénévoles portant le sigle de l'association pour gérer les flux de véhicules, les orienter vers des parkings libres et sécuriser le flux des piétons sur les passages cloutés. En plus, trois entreprises de la zone industrielle des Doucettes mettent leur parking à disposition pour les grands événements. On est bien loin ici de l'islam des caves qui a marqué Garges juste après la construction des grands ensembles dans les années 1970. Un ancien Africain résidant dans un foyer de travailleurs migrants rapporte qu'à l'époque, il y avait une salle de prière dans une cave d'immeuble située à côté de la mairie, et une autre à l'intérieur du foyer. Il n'y avait pas d'imam fixe : "C'est celui qui est le mieux d'entre nous qui dirige la prière. Mais quand il y a une mosquée, oui, il faut un imam ; mais dans les salles de prière, il n'y a pas d'imam. $»^{14}$

La nouvelle mosquée pakistanaise de Garges est implantée dans un vaste pavillon moderne rue Danielle Casanova (figure 5). Contrastant fortement avec les premières implantations musulmanes d'origine maghrébine, cette mosquée traduit bien le nouvel âge de l'islam dans les banlieues. Le premier âge, l'islam des Darons, décrit par Gilles Kepel, qui s'accordait avec la soumission des pères ou des grands-pères souvent illettrés, est dépassé - de même que le second âge, celui des frères et des blédards qui étaient détenteurs d'un projet politique et qui revendiquaient le voile pour les femmes (Kepel, 2012). L'islam d'aujourd'hui est porté par les enfants de la seconde et de la troisième génération, sinon 
par des migrants comme les Turcs et les Pakistanais aux assises financières solides et fortement soutenus par leur pays d'origine. Sa place nouvelle se traduit par la qualité des implantations cultuelles comme on le voit sur la photographie. Et une bonne partie des revendications se focalise désormais sur le halal, sinon sur des projets d'extension susceptibles de conduire à des conflits avec les municipalités concernées.

Figure 5: La mosquée pakistanaise de Garges aménagée dans un pavillon

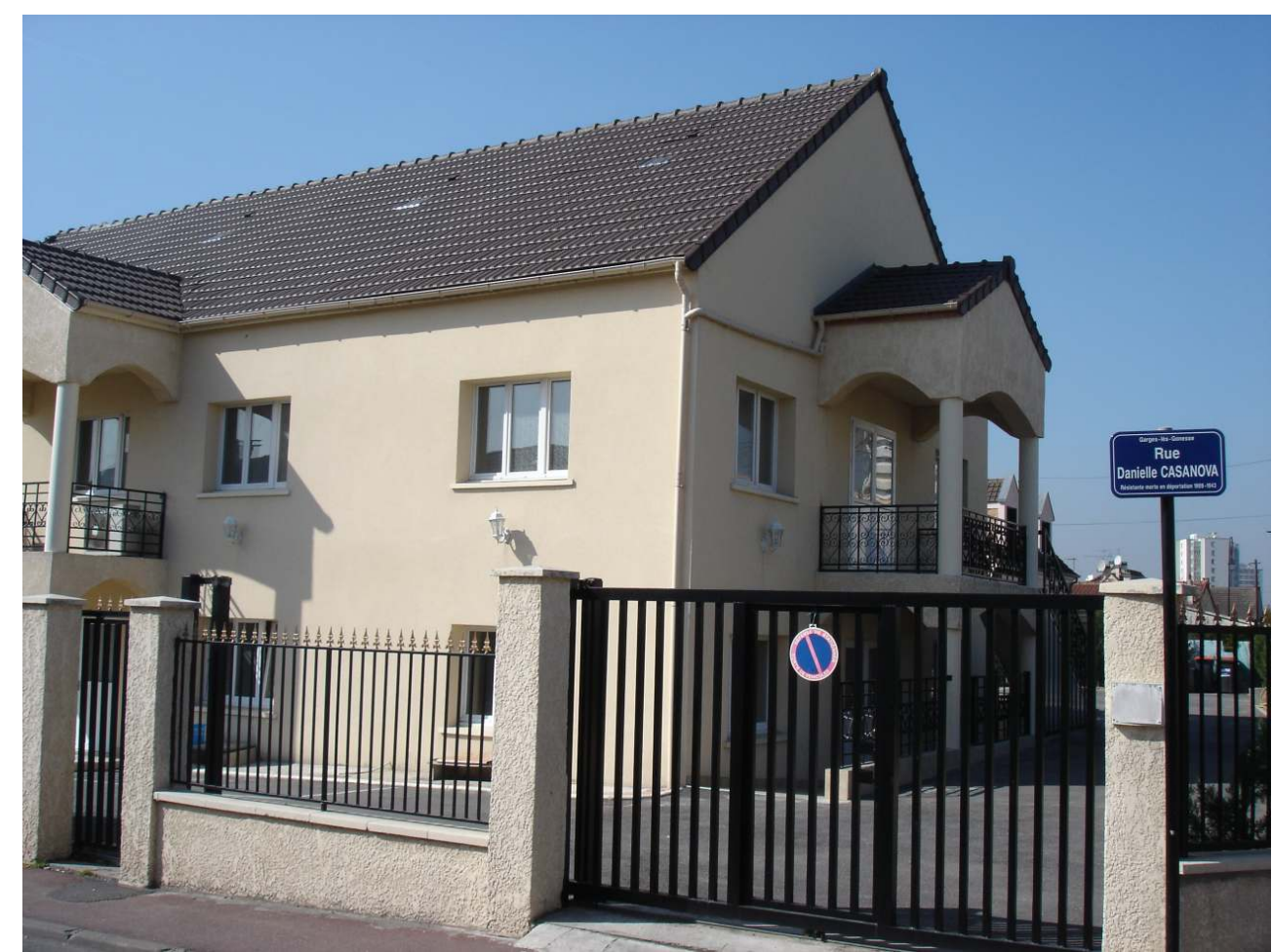

cliché : Hervé Vieillard-Baron, 2012

Localement, les accommodements concernant le garage des véhicules des fidèles sont loin d'être purement anecdotiques. En effet, plusieurs permis de construire ont été refusés à cause de l'insuffisance de places de parking dans les projets. Prétexte ou non, le sujet reste sensible pour les municipalités, même si celles-ci ont appris à mieux le gérer au fil du temps. Après discussions sur les parkings, des autorisations ont été données pour la construction d'une église chaldéenne à Arnouville et d'une mosquée dans le grand ensemble de la Muette à Garges. Certains terrains ont été vendus à l'euro symbolique (par exemple pour la construction d'une mosquée à Sarcelles), d'autres au prix des domaines (comme à Villiers-le-Bel); d'autres encore ont pu bénéficier (comme à Garges) d'un bail emphytéotique ou d'une opération démolition-reconstruction. Si l'on met à part Gargeslès-Gonesse où la municipalité UMP est en conflit ouvert avec deux mosquées, force est de constater que bien souvent, l'opposition aux implantations nouvelles vient davantage du voisinage que des pouvoirs publics. Des associations de défense ont pu se constituer provisoirement. Les anciens chrétiens ont parfois le sentiment d'être débordés par ces nouveaux pratiquants issus de tous les pays du monde et aux manifestations souvent expansives. 


\section{Une ouverture très variable sur la société locale} ont fait le pari de l'ouverture vers la société locale, si l'on en croit le discours des
principaux responsables. Il s'agit non seulement des paroisses catholiques et protestantes réformées, mais aussi de certaines associations musulmanes. Le cas de l'association des Français musulmans (AFMV) de Villiers-le-Bel est significatif à cet égard ${ }^{15}$. Son histoire prend forme en 1981 avec la fondation de l'association indo-vietnamienne de Villiers-leBel, composée uniquement d'Asiatiques à l'origine. Une première ouverture s'opère avec l'intégration de musulmans maghrébins dans les années qui suivent, puis avec le changement de nom de l'association (AFMV). Des Pakistanais s'y associent provisoirement; mais, tout en conservant des rapports cordiaux, ces derniers ont finalement décidé de créer leur propre mosquée notamment pour des raisons linguistiques, le prêche en pakistanais devant s'imposer aux natifs du Pakistan et à leurs enfants pour les responsables communautaires. Il faut rappeler aussi que l'islam «tamoul» est caractérisé par un rite spécifique, le rite chaféite, et une influence non négligeable de l'hindouisme (Delval, 1998).

Apparemment, les anciens de l'association des Français musulmans ont su nouer de bonnes relations avec les plus jeunes et avec l'église catholique locale. Toujours selon Farouk Yagoubi, ils auraient accepté des formes de modernisation dans les pratiques en se détachant progressivement des pays d'origine et en acceptant l'affiliation à un islam de France autonome, ouvert aux cultures extérieures et largement apolitique, dans un souci de transparence... Les deuxième et troisième générations, nées en France, auraient joué un rôle important en ce sens. Parallèlement, les relations avec la ville se sont renforcées. L'association participe depuis une dizaine d'années aux fêtes de quartiers, aux manifestations municipales et aux forums des associations. De son côté, la municipalité n'hésite pas à prêter un gymnase à l'occasion de l'Aïd; celui-ci regroupe alors plus de 3000 personnes venues de toute la région. Par ailleurs, l'association dispose de deux types de locaux à Villiers-le-Bel : le rez-de-chaussée d'un immeuble, dénommé «la mosquée ", situé dans le grand ensemble " Derrière-les-murs-de-Monseigneur », et un centre culturel localisé rue d'Hérivaux. Pendant la pause déjeuner du vendredi, il n'est pas rare que des salariés des entreprises locales se rendent à la mosquée pour prier.

Enfin, outre ses activités strictement cultuelles, l'association développe des activités éducatives - cours d'arabe, éducation religieuse, soutien scolaire (6 à 700 enfants seraient concernés chaque semaine les mercredi, samedi et dimanche), alphabétisation des adultes - mais aussi diverses activités sportives. Des rencontres régulières sont organisées mêlant hommes et femmes d'âges variés: conférences mensuelles ou dîners débats sur des thèmes divers : islam, réussite des enfants, origine de l'homme... Des rencontres assorties d'une table ronde sont organisées de temps à autre sur un sujet porteur. La dernière a eu lieu le 9 avril 2011 à l'Espace Marcel Pagnol (la plus grande salle de la ville) sur le thème : «Islam, science et modernité». Annoncée par l'agence internationale de Presse coranique, elle a accueilli diverses manifestations annexes dont certaines avaient un caractère radical et propagandiste : chants, stands de livres et cédéroms, prêt-à-porter féminin, espace dédié aux fatwas, exposition de l'IMA sur les sciences arabes. 


\section{Des appartenances mieux reconnues?}

39 transformé le paysage urbain et social de la communauté d'agglomération Val de France. La diversité des édifices religieux et la pluralité des identités culturelles, associée à la multiplicité des origines migratoires avec toutes les formes de syncrétisme qui en résultent, reposent la question de l'adaptation de la société locale et, plus largement, celle de la tolérance à l'autre. Il va de soi que les pratiques religieuses et le souci, inégalement partagé, de s'affirmer dans l'espace public peuvent entrer en tension avec les usages traditionnels et les logiques sociales qui s'inventent dans la ville.

Au-delà de la position de stricte neutralité imposée aux pouvoirs publics par la loi de 1905, les circonstances conduisent les interlocuteurs institutionnels à se placer en position de négociation. Les collectivités territoriales les plus concernées par l'immigration ont tendance à trouver des accommodements raisonnables, en permettant la construction d'édifices religieux dignes et fonctionnels, et en participant au financement de centres culturels. De leur côté, les paroisses catholiques ont appris à négocier avec les chrétiens nouvellement arrivés dans le cadre de la Pastorale des migrants ; elles ouvrent leurs locaux et adaptent leur liturgie. En retour, la présence active des migrants contribue puissamment au renouveau des pratiques. L'église catholique Jean XXIII construite au cœur du grand ensemble de Sarcelles a été agrandie en 2003 : tout un symbole puisque deux églises catholiques y avaient été fermées dans les années 1970.

Parallèlement, ce nouveau dynamisme religieux ne cesse d'alimenter le débat sur l'actualité du modèle français de laïcité et ses effets, alors même qu'une partie des musulmans considère que la laïcité a détruit le christianisme, longtemps perçu comme majoritaire. Les relations très sensibles entre « religion et vie publique ", et « religion et école » sont au centre des débats et conduisent souvent à des formes de compromis, si ce n'est à des polémiques plus ou moins attisées par le voisinage et par des personnalités politiques qui se situent à droite, voire à l'extrême droite de l'échiquier politique.

visibilité des signes religieux et l'effervescence des pratiques ont profondément modifié le statut de la religion dans l'espace public de la banlieue, peut-on dire pour autant que les cultures d'origine des migrants et leurs anciennes appartenances sont mieux acceptées que par le passé ? Peut-on dire, de l'autre côté, que l'acculturation progresse de manière positive? Sans doute une génération de conseillers municipaux originaires du Maghreb, du Moyen-Orient et d'Afrique subsaharienne émerge-t-elle dans certaines communes, comme Sarcelles ou Villiers-le-Bel. Il n'en reste pas moins que la très faible représentation des Français issus de l'immigration dans la représentation nationale, à l'Assemblée nationale comme au Sénat, constitue une sorte d'anachronisme dans l'espace politique contemporain. Faut-il alors s'étonner des formes de substitution qui conduisent les plus actifs, ou les plus révoltés, à s'investir dans le religieux? 


\section{BIBLIOGRAPHIE}

Arkoun M. (dir.), (2009), Histoire de l'Islam et des musulmans en France, du Moyen-Age à nos jours, Albin Michel.

Azria R. et Hervieu-Leger D. (dir.), (2010), Dictionnaire des faits religieux, Paris, PUF.

Benveniste A. (1999) «Territorialisation et circulation des échanges sociaux dans deux sites de la banlieue parisienne », Journal des anthropologues, $\mathrm{N}^{\circ}$ 77-78, pp 257-273

Dejean F. (2010) Les Dimensions spatiales des Eglises évangéliques et pentecôtistes dans une commune de banlieue parisienne (Saint-Denis) et dans deux arrondissements montréalais (Rosemont et Villeray), Thèse de doctorat, Université de Paris Ouest-Nanterre-La Défense et INRS-UCS, 385p.

Delval R. (1998) Musulmans français d'origine indienne : Réunion, France métropolitaine, anciens établissements français de l'Inde, Paris, Publication du Centre des Hautes études sur l'Afrique et l'Asie modernes (CHEAM), 170p.

Endelstein L., Fath S. et Mathieu S. (ed.) (2010) Dieu change en ville. Religion, espace, immigration, Paris, L'Harmattan, 222p.

Fath S., (2004), Militants de la Bible aux Etats-Unis, Evangéliques et fondamentalistes du Sud, Paris, Autrement

Gremion C. (2012), Les religions dans la ville d'aujourd'hui, L'Harmattan.

Hervieu-Leger D., La religion en miettes ou la question des sectes, Paris, Calmann-Lévy, 2002

Kepel G. (2012), Quatre-Vingt-treize, Paris, Gallimard, 336p.

Kepel G. (2012), Banlieue de la République, Gallimard.

Podselver L. (2000) « De la périphérie au centre : Sarcelles, ville juive », in Bordes-Benayoun C. (dir.) Les juifs et la ville, Toulouse, Presses universitaires du Mirail, pp. 79-90.

Roy O., (2005), L'islam globalisé, Paris, Le Seuil,

Tribalat M. (2006) « Les concentrations ethniques en France », http://www.societe-destrategie.asso.fr/pdf/agir28txt4.pdf, 10p.

Vieillard-Baron H. (1992), « De l'exil aux logiques d'enracinement : l'exemple de Sarcelles », in Ferréol G. (dir.) Intégration et exclusion, Lille, Presses universitaires de Lille, pp. 105-128

\section{NOTES}

1. La première création issue d'une migration étrangère est ici l'église arménienne apostolique Sainte-Croix de Varak inaugurée à Arnouville en 1932 par l'archevêque de l'Eglise arménienne de Paris. C'est une église d'apparence classique construite par l'architecte Armen Agopian, avec un clocher octogonal de style arménien; elle comprend aujourd'hui dans la cour un khatchkar, commémorant les victimes du génocide. On comptait 344 Arméniens au recensement de 1926 à Arnouville, 698 à celui de 1931, sans compter leurs enfants nés en France. Il s'agit du troisième regroupement de la région parisienne, après Alfortville et Issy-les-Moulineaux. 
2. INSEE, recensement partiel de 2008, base de données harmonisées. En 2010, on comptait 58614 habitants à Sarcelles, 40012 à Garges, 26736 à Villiers-le-bel et 13538 à Arnouville.

3. En 1994, un recensement exhaustif des titres de séjour mené avec le commissariat de Sarcelles y soulignait la présence de 92 nationalités.

4. On désigne par là les jeunes étrangers et les jeunes Français de moins de 18 ans nés en France d'un parent ou d'un grand parent né à l'étranger et ayant immigré en France.

5. Selon le pasteur Daniel Liechti, vice-président du Conseil national des évangéliques de France, le protestantisme évangélique représenterait 470000 pratiquants réguliers en 2012, soit un bon tiers des protestants de France et trois-quarts des pratiquants. Une nouvelle église évangélique locale naîtrait ainsi tous les dix jours en France... En nombre, on trouve d'abord les Assemblées de Dieu (391 Eglises), la mission évangélique tsigane (218 églises), la Fédération des Eglises évangéliques baptistes (120 Eglises) et les Communautés et assemblées évangéliques de France (Journal La Croix, 23/01/2013). En Ile-de-France, le taux de pénétration le plus élevé se situe en Seine-Saint-Denis, dans le sud-est du Val d'Oise et dans l'Essonne.

6. Le décompte des oratoires juifs est particulièrement difficile étant donné leur peu de lisibilité dans l'espace (appartements, locaux résidentiels, ancienne boutique, etc..). De plus, une partie d'entre eux résulte de fractionnements communautaires qui peuvent s'avérer provisoires. Par exemple à Sarcelles, on relève en 2012, outre la grande synagogue consistoriale, une bonne quinzaine de synagogues, certaines d'entre elles portant le nom d'un grand rabbin : Beth Avraham, Beth Ephraim, Beth Habad, Beth Hacohanim, Beth Hamidrach, Beth Menahem, la Ghriba, Hag Israel, Heder Tachbar, Ismah Israel, Net's ha'hama, Rav Afriat, Rav Israel, Ouaknine Meyer et la synagogue du secteur pavillonnaire des Chardonnerettes.

Interviewé par Claude Jannoud et Marie-Hélène Pinel pour leur ouvrage " La première ville nouvelle", paru en 1974 au Mercure de France, le rabbin Guedj de Sarcelles évoque une synagogue et deux oratoires. Pour la communauté juive des trois villes de Sarcelles, Garges et Villiers-le-Bel, il évoque à l'époque la composition suivante : « $35 \%$ d'Algériens, $35 \%$ de Tunisiens, $12 \%$ d'Egyptiens et $5 \%$ de gens de France ».

7. Situé au Nord de Paris, ce réseau est affilié à la Fédération protestante de France et au Conseil national des Eglises évangéliques dont le siège est à Bagneux. Il fait partie des «Assemblées de Dieu » et il possède des implantations actives dans les communes de Bobigny, Saint-Denis, Chambly, Goussainville, Sarcelles, Persan, Survilliers-Saint Witz. Selon le principe des "Assemblées de Dieu», chaque église constitue une association cultuelle autonome avec des financements propres.

Ce réseau comprend un institut biblique et théologique, et accueille une église destinée aux malentendants.

8. Site louyehi.wordpress.com consulté en juin 2011.

9. On trouve presque tous les courants de l'islam dans la communauté d'agglomération, des plus modérés aux plus radicaux, la mosquée El Irshad de Garges ayant accueilli notamment des Frères musulmans et des Salafistes.

10. Il s'agit par exemple à Garges les Gonesse de l'Association cultuelle des musulmans de Garges (ACMG), de « Foi et Unicité », de l'association islamique des Français de l'Inde et de l'Indochine, de l'association amicale des travailleurs turcs et ses sympathisants de Garges et des environs (AATTSGE), de l'association pakistanaise Falah-e-Darain.

11. Cette mosquée, située avenue Paul Vaillant Couturier à Garges, est localisée tout près de la zone industrielle des Doucettes, à proximité du quartier de grands ensembles du même nom. Elle est gérée par l'association cultuelle des musulmans de Garges (ACMG). Cette dernière ne se confond pas avec l'association islamique et culturelle de la Muette (rue Paul Langevin) ni avec l'association cultuelle, culturelle des Musulmans de Garges-lès-Gonesse.

12. Le Conseil national des églises évangéliques de France (CNEF) a été créé justement pour mettre un peu d'ordre dans ce foisonnement et éviter les confusions avec certains groupes 
sectaires dominés par des pasteurs qui profitent financièrement de la crédulité de leurs fidèles tout en se revendiquant évangéliques, sans avoir pour autant de formation théologique.

13. Le Journal officiel des associations, consultable sur Internet, a enregistré entre avril 2003 et janvier 2012, rien que sur la ville de Sarcelles, 23 associations à caractère cultuel dont 18 créations, 4 modifications de nom ou de statut, et une dissolution (l'Action évangélique "Pain aux hommes »). Pour les créations, il s'agit de l'association culturelle et cultuelle chrétienne de l'église de Dieu en Christ de France, de la mission d'évangélisation mondiale «La parole agissante ", de l'Eglise du christianisme céleste - paroisse Saint-Samuel, de l'Eglise chrétienne évangélique (Berée), de l'Eglise du christianisme céleste "Paroisse Jérusalem céleste», de la «Paroisse Carmel», de l'Eglise évangélique baptiste "l'Espérance divine », de la Communauté évangélique "Grain de Sénevé », du Centre chrétien "Viens et Vois », de l'Eglise évangélique de la nouvelle naissance, de l'Assemblée "Feu apostolique de Christ», de la Mission évangélique "Viens et Vois», de l'association chrétienne «Souffle de vie S.V.», de la Mission évangélique baptiste Rédemption internationale, de l'Eglise du christianisme céleste - Le mont Sinaï, de l'Eglise évangélique sentinelle vigilante de Jésus-Christ en France, de l'Eglise évangélique «Tous ensemble », et enfin de l'Eglise évangélique « Mission internationale La foi en action ».

14. Collecte de témoignages des Archives municipales, mars 2000.

15. Les informations données ici résultent de la transcription de l'entretien de Catherine Roth avec Farouk Yagougi, un des responsables de l'association (mars 2012).

\section{RÉSUMÉS}

Les grandes vagues migratoires du 20ème siècle ont suscité la création de nombreux lieux de culte : églises d'Orient, synagogues, mosquées, pagodes, temples évangéliques... Au sein de l'espace urbain, certains d'entre eux affichent leur caractère religieux ; d'autres restent invisibles, implantés dans des locaux détournés de leur fonction initiale. Dans les pratiques, on constate différentes formes de recomposition des anciennes appartenances, mais aussi le souci de valoriser les identités d'origine.

Dans cet article, l'auteur montre, à partir de l'exemple significatif de la communauté d'agglomération "Val de France" (Sarcelles, Villiers-le-Bel, Garge-lès-Gonesse, Arnouville) que le pluralisme religieux est devenu une donnée de base de la banlieue parisienne. En une centaine d'années, on est passé de quatre à soixante-neuf implantations cultuelles dans le cadre des quatre communes étudiées.

Avec le temps, chaque lieu de culte tend à devenir non seulement un espace de ressourcement communautaire, mais aussi un centre de vie sociale et culturelle. Les questions concernant l'agrandissement des locaux et le voisinage deviennent par conséquent des questions cruciales dans la gestion de l'espace communal. Mais en dépit de tensions multiples, l'auteur constate que les acteurs concernés se placent plutôt en position de négociation dans un esprit de tolérance.

\section{INDEX}

Thèmes : Carnets de recherches

Mots-clés : église, banlieue, communautarisme 


\section{AUTEUR}

\section{HERVÉ VIEILLARD-BARON}

\section{Géographe}

Université Paris Ouest Nanterre

herve.vieillard-baron@wanadoo.fr 